\title{
To do or not to do lymphadenectomy in part-solid lung adenocarcinoma
}

\author{
Woohyun Jung, Sukki Cho \\ Department of Thoracic and Cardiovascular Surgery, Seoul National University Bundang Hospital, Seongnam-si, Gyeonggi-do, Republic of Korea \\ Correspondence to: Sukki Cho, MD, PhD. Department of Thoracic and Cardiovascular Surgery, Seoul National University Bundang Hospital, 166, \\ Gumi-ro, Bundang-gu, Seongnam-si, Gyeonggi-do 463-707, Republic of Korea. Email: skcho@snubh.org. \\ Provenance: This is an invited Editorial commissioned by the Section Editor Feichao Bao (Department of Thoracic Surgery, The First Affiliated \\ Hospital, Zhejiang University, Hangzhou, China). \\ Comment on: Hattori A, Matsunaga T, Takamochi K, et al. Significance of Lymphadenectomy in Part-Solid Lung Adenocarcinoma: Propensity Score \\ Matched Analysis. Ann Thorac Surg 2018. [Epub ahead of print].
}

Submitted Aug 14, 2018. Accepted for publication Sep 13, 2018.

doi: $10.21037 /$ jtd.2018.09.76

View this article at: http://dx.doi.org/10.21037/jtd.2018.09.76

Dr. Hattori and his colleagues recently published an article titled, "Significance of Lymphadenectomy in PartSolid Lung Adenocarcinoma: Propensity Score Matched Analysis" in The Annals of Thoracic Surgery. They reported the clinical outcomes, according to the type of lymph node dissection (LND) in patients with clinical stage I, part-solid, lung adenocarcinoma. The type of LND was shown to have no influence on the prognosis in both non-solid, and soliddominant lesions, which was supported by a propensityscore analysis. Based on the results, they concluded that the mediastinal LND (m-LND) could be selectively omitted in some patients based on preoperative parameters, such as carcinoembryonic antigen (CEA) or their maximum standardized uptake value (SUVmax).

The goal of lung cancer surgery is to achieve local control and confirm disease extent. As such, appropriate determination of the extent of lung excision and LN dissection is important. The anatomical extent of disease should be well predicted based on clinical information, such as tumor size, SUVmax, or CEA. If such a prediction is not possible, performing a resection could still result in satisfactory outcome for any stage lung cancer. As such, there has been much effort to determine the conditions surrounding a limited resection of the lung, especially for a part-solid adenocarcinoma $(1,2)$. A randomized controlled study was performed by the Lung Cancer Study Group, comparing the outcomes of lobectomy and limited pulmonary resection. They demonstrated that a limited pulmonary resection did not produce any improvements in perioperative morbidity and mortality; however, they did show that it may be associated with higher death rate and a locoregional recurrence rate (3). Recent developments of imaging modalities had brought improvements for the detection of small-sized non-small cell lung cancer (NSCLC), and with it, brought discussion about the usefulness of a limited resection. The part-solid adenocarcinoma, which is known to have a better prognosis than a pure solid tumor, was considered a good candidate for the limited pulmonary resection. To address this issue, randomized and controlled trials which peripherally located NSCLCs with $2 \mathrm{~cm}$ or less in size, were conducted by the Cancer and Leukemia Group B, (CALGB 14053) in the United States, and by the Japan Clinical Oncology Group (JCOG 0802) in Japan, with a large sample size (target accrual numbers were 1,297 and 1,100 patients, respectively).

Whether to perform a systemic m-LND for NSCLC remains controversial. Proponents emphasize that it is more accurate with the respect to staging, than the limited LND. In regards to the therapeutic effect, it has been reported that $\mathrm{m}$-LND increased chance of survival of the patient, by removing the occult N2 disease (4). Conversely, opponents of $\mathrm{m}$-LND have asserted that it could potentially result in more adverse events, such as bleeding, chylothorax, or longer hospitalization. Similar to the discussion of resection, the introduction of a part- 
solid lung adenocarcinoma fueled a debate about the necessity of m-LND. Before debating, we have to know the exact rate of which there is nodal involvement in partsolid lung adenocarcinoma. The authors of this article previously reported the clinicopathologic characteristics of clinical stage 1A adenocarcinoma, and rate of both of $\mathrm{pN} 1$ and $\mathrm{pN} 2$ metastasis were reported at 2\% (5). Yoshida et al. reported that $\mathrm{N} 0$ was proven that after a $\mathrm{m}-\mathrm{LND}$, in all patients with non-solid lung adenocarcinoma, only $1 \%$ of patients with part-solid lung adenocarcinoma had node metastasis (6). In addition, Ye et al. (7) reported that among patients with clinical stage $1 \mathrm{~A}$, part-solid lung cancer, $\mathrm{pN} 1$ was observed at $2 \%$ and $\mathrm{pN} 2$ at $1 \%$. Since the entire studied population underwent m-LND testing, the results are more meaningful and noteworthy. Based on the above findings, some insisted that a m-LND may not be necessary for lung adenocarcinoma with a non-solid nodule as well as for some with a part-solid nodule. Murakawa and colleagues reported that recurrence-free survival was solely dependent on the solid component size, and suggested that a $\mathrm{T}$ factor measured using only the solid component, may be a more accurate prognostic guideline (8). Burt and colleagues likewise, showed that the maximum solid diameter had been associated with an overall increased rate of survival in a largely non-Asian cohort (9).

Two methods have been mainly used to evaluate the extent of ground glass opacity for a part-solid adenocarcinoma: the consolidation tumor ratio (CTR) from the lung window, and the tumor disappearance ratio (TDR) from the mediastinal window. Higher specificity was obtained from using the CTR, while higher sensitivity was obtained using a TDR. Therefore, the TDR method, in the case of a more invasive cancer, would likely be misdiagnosed as radiologically noninvasive, whereas CTR provided a clinically safe criteria to identify a noninvasive cancer (10). Okada and colleagues revealed that adenocarcinoma, with high SUVmax and low GGO ratio is more closely associated with lymphatic, vessel, pleural invasion, LN metastasis, and recurrence (11).

In this regard, the study by Hattori and colleagues, focused on investigating the effect of m-LND on the survival rate in stage I part-solid adenocarcinoma according to CTR. The study enrolled 462 patients. Hilar LN dissection (h-LND) was performed on $32 \%$ of the patients, based on the results of intraoperative frozen-section diagnosis of the hilar LN. They showed that none of the stage I, part-solid lung adenocarcinoma had a LN metastasis when it was GGO dominant, (CTR less than 0.5) and there was the possibility of setting up the prediction model for the pathologic LN status, according to preoperative clinical information, such as SUVmax and CEA, in patients with a solid-dominant tumor (CTR more than 0.5). This study, given this finding, deserves much praise, despite the limitations of retrospective studies. Finally, they concluded that there was no difference in survival outcome between the m-LND group and h-LND group. To develop a standardized guideline for LN dissection in lung cancer, the LN status prediction model. A model based on preoperative clinical information, such as tumor size, CTR on part-solid nodules, tumor location, and SUVmax, should be developed, as attempted by the authors of this article. Moreover, it needs to be verified through further, randomized controlled trials. This would be the only way to determine the necessity of $\mathrm{m}$-LND.

\section{Acknowledgements}

None.

\section{Footnote}

Conflicts of Interest: The authors have no conflicts of interest to declare.

\section{References}

1. Suzuki K, Asamura H, Kusumoto M, et al. "Early" peripheral lung cancer: prognostic significance of ground glass opacity on thin-section computed tomographic scan. Ann Thorac Surg 2002;74:1635-9.

2. Hattori A, Suzuki K, Matsunaga T, et al. Is limited resection appropriate for radiologically "solid" tumors in small lung cancers? Ann Thorac Surg. 2012;94:212-5.

3. Ginsberg RJ, Rubinstein LV. Randomized trial of lobectomy versus limited resection for T1 N0 non-small cell lung cancer. Lung Cancer Study Group. Ann Thorac Surg 1995;60:615-22; discussion 622-3.

4. Gajra A, Newman N, Gamble GP, et al. Effect of number of lymph nodes sampled on outcome in patients with stage I non-small-cell lung cancer. J Clin Oncol 2003;21:1029-34.

5. Hattori A, Matsunaga T, Takamochi K, et al. Importance of Ground Glass Opacity Component in Clinical Stage IA Radiologic Invasive Lung Cancer. Ann Thorac Surg 2017;104:313-20.

6. Yoshida J, Nagai K, Yokose T, et al. Primary peripheral lung carcinoma smaller than $1 \mathrm{~cm}$ in diameter. Chest 
1998;114:710-2.

7. Ye B, Cheng M, Li W, et al. Predictive factors for lymph node metastasis in clinical stage IA lung adenocarcinoma. Ann Thorac Surg 2014;98:217-23.

8. Murakawa T, Konoeda C, Ito T, et al. The ground glass opacity component can be eliminated from the T-factor assessment of lung adenocarcinoma. Eur J Cardiothorac Surg 2013;43:925-32.

9. Burt BM, Leung AN, Yanagawa M, et al. Diameter of Solid Tumor Component Alone Should be Used to Establish T Stage in Lung Adenocarcinoma. Ann Surg Oncol 2015;22 Suppl 3:S1318-23.

10. Suzuki K, Koike T, Asakawa T, et al. A prospective

Cite this article as: Jung W, Cho S. To do or not to do lymphadenectomy in part-solid lung adenocarcinoma. J Thorac Dis 2018;10(Suppl 33):S3899-S3901. doi: 10.21037/ jtd.2018.09.76 radiological study of thin-section computed tomography to predict pathological noninvasiveness in peripheral clinical IA lung cancer (Japan Clinical Oncology Group 0201). J Thorac Oncol 2011;6:751-6.

11. Okada M, Nakayama H, Okumura S, et al. Multicenter analysis of high-resolution computed tomography and positron emission tomography/computed tomography findings to choose therapeutic strategies for clinical stage IA lung adenocarcinoma. J Thorac Cardiovasc Surg 2011;141:1384-91.

(English Language Editor: Jeremy Dean Chapnick, AME Publishing Company) 